\title{
Über das Zentralnervensystem des Skorpions und der Spinnen.
}

Ein zweiter Beitrag zur Stammesgeschichte der Arachnoiden.

Von

B. Haller.

Hierzu Tafel XXVI und 3 Textfiguren.

In einer unlängst im selben Bande dieses Archivs erschienenen Abhandlung (4) zeigte ich, dass die Atmungsorgane, speziell die sogenannten Lungen der Arachnoiden nicht wie die RayLankestersche Theorie (7) verlangt, von den Kiemen des Limulus ableitbar seien, sondern dass vielmehr jene Organe aus Tracheenbüscheln sich entfaltet haben, wie dies schon Leuckart behauptet hatte. Soweit habe ich mit anderen die Berechtigung der Ableitung der Arachnoiden von Xyphosuren abgesprochen. Doch obgleich bei der Beurteilung phyletischer Fragen der Arachnoiden die Atmungsorgane in erster Linie dazu berufen sind, ein entscheidendes Wort abzugeben, müssen daneben auch andere Organsysteme in Betracht kommen. Dies war ja schon RayLankester (8) in seiner zweiten diesbezüglichen Abhandlung klar, obgleich in jener Schrift Konvergentes phyletische Bewertung erfuhr.

Seit Viallanes (9) wissen wir, dass die pilzhutförmigen Kōrper, die Globuli, im Cerebralganglion bei Limulus eine so hohe Entfaltung erfahren haben, ein so măchtiges Faltensystem darstellen, wie sie unter den Gliedertieren, bei denen sie sich eben finden, selbst denjenigen unter ihnen, welche die höchste Entfaltung dieser Hirnteile infolge ihrer hohen Intelligenz erfahren haben, den Hymenopteren und unter diesen selbst bei der Hornisse nicht erreicht ist.

Sollte also jene Verwandtschaft zwischen Limulus und den Skorpionen - trotz des Gegenbeweises durch das Verhalten der Atmungsorgane - doch bestehen, so müssten letztere entweder eine gleich hohe Entfaltung ihrer Globuli aufweisen wie Limulus, oder im Falle bei den Skorpionen irgend aus einem Grunde ein Rückbildungsprozess jener Hirnteile eingetreten wäre, müssten hierfür die Strukturverhältnisse einstehen. Für einen solchen 
Rückbildungsprozess liegt allerdings gar kein Grund vor, denn wo bei Tracheaten oder Branchiaten ein Rücktritt der Globuli sich zeigt, handelt es sich stets um die Folgen starken Parasitismus ihrer Träger, oder um die Kompensation durch die mächtig entfalteten Komplexaugen und ihrer Ganglien.

Es müsste also bei dieser Fragestellung gezeigt werden, dass entweder die Skorpione einen diesbezüglichen Rückbildungsweg durchmachten, oder sie bezüglich der Entfaltung der Globuli dem Limulus mehr oder weniger gleichgestellt sind. Es könnte ja dann dieser Prozess sich auf Skorpione beschränken (?) und würden dann Araneen bezüglich der Globuli immerhin noch eine hohe Stellung einnehmen.

Es war somit diese Fragestellung, welche vorliegende kleine Schrift veranlasste, allein bei der grossen Unkenntnis der gesamten Verhältnisse des Zentralnervensystems der höheren Arachnoiden -- denn ausser der Beschreibung Börners über jenes der Pedipalpen (1) und die recht kurze Janecks (5) über jenes der Spinnen, ist in der Literatu: nichts vorhanden - war es geboten, auch das gesamte Zentralnervensystem einigermassen zu berücksichtigen. Dabei lag es mir fern, eine ausführliche Verfolgung dieses Themas vorzunehmen.

Für die Spinnen begnügte ich mich fast ausschliesslich mit den Verbältnissen der grosses Material bietenden Kreuzspinne, da ja das Zentralnervensystem unter den Spinnen doch nur untergeordnete, ganz unwesentliche Unterschiede aufweist. Von Skorpionen untersuchte ich aus gleichem Grunde - und da mir auch kein weiteres Material zur Verfügung stand - Scorpio europaeus. Dabei musste in beiden Fällen der Topographie halber das Gefässsystem einigermassen berücksichtigt werden.

\section{A. Scorpio europaeus.}

Bekanntlich besteht das Zentralnervensystem des Skorpions aus einem konzentrierten Abschnitt im cephalothorakalen Teil des Körpers, der aus fünf miteinander engverschmolzenen Ganglienpaaren, entsprechend den fünf Beinpaaren (Kieferfuss mitgerechnet) und dem Supraösophagealganglion oder dem Gehirn besteht. Das Bauchmark verlängert sich dann als Ganglienkette in das Abdomen; dieser Teil des Zentralnervensystems bleibt in vorliegender Schrift unberïcksichtigt. 
Die beiden Cerebralganglien (Textfig. $1 \mathrm{~A}, \mathrm{Cg}$ ) sind zwar dorsalwärts gewölbt, so dass die Paarigkeit des Gehirns sofort erkenntlich ist, allein die beiden Hälften sind medianwärts miteinander so eng verwachsen, dass eine Querverbindung unter ihnen äusserlich nicht erkenntlich ist (Textfig. 2). Sie sind jedes für sich mit dem Bauchmark (Textfig. $1 \mathrm{~A}, \mathrm{bm}$ ) eng verbunden, so den Ösophagus umgreifend, doch ist auch diese Verbindung keine rein kommissurale mehr, da sie bei der grossen Konzentration des vorderen Abschnitts vom Zentralnervensystem, von einer vielschichtigen Ganglienzellage überzogen ist. An dem oralwärtigen Teil dieser Verbindung zeigt sich eine gangliöse Verdickung (g), aus der ein starker Nerv entspringt, welcher in senkrechter Lage am oralen Rande des Gehirns jederseits hinaufzieht, um sich dann am dorsalen Rande des Gehirns in zwei Äste zu teilen. Der eine (on) Ast gelangt in die Oberlippe, wie das periphere Verhalten dieses Nervens schon $\mathrm{New}$ port (6) richtig dar-

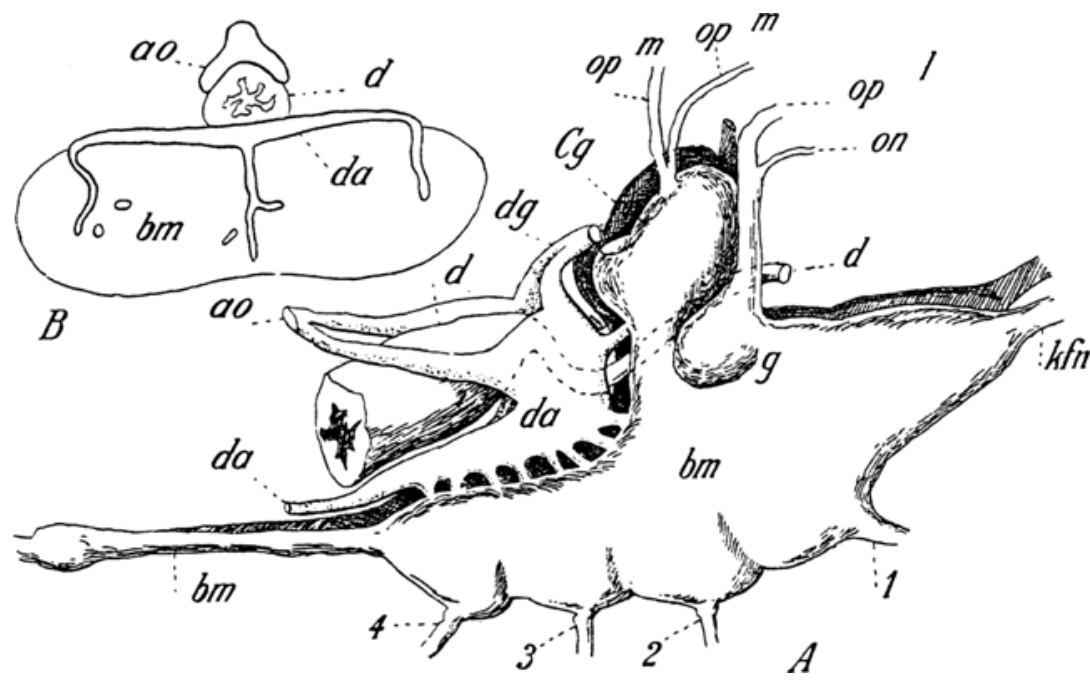

Fig. 1.

Scorpio europa eus. A. Das cephalothorakale Zentralnervensystem von der rechten Seite, samt dem Darm (d) und seinem Blutgefässsystem. ao = Aorta, $\mathrm{dg}==$ Dorsalgefäss des Gehirns ; da $=$ dorsale Arterie ; $\mathrm{Cg}=$ Cerabralganglion ; opm = Optici der Medianaugen; opl = desgleichen der Lateralaugen; on = Oberlippennerv; $\mathrm{g}=$ dessen Ganglion; $\mathrm{bm}=$ vorderes, $\mathrm{bm}=$ ein Teil des hinteren Bauchmarkes; $k \mathrm{fn}=$ Kieferfussnerv $; 1-4=$ die Nerven der vier übrigen Beinpaare. B. Querschnitt durch das vordere Bauchmark (bm), um das Verhalten der Dorsalarterie zu zeigen. $\mathrm{d}=$ Darm; a $_{0}=$ Aorta. 
gestellt hatte, der andere ist der Nerv der Seitenaugen (op. l). Dieser letzte Nerv entspringt indessen, wie wir bei der Spinne sehen werden, nicht aus jenem Ganglion (g), dieses gehört vielmehr nur dem Oberlippennerv an, sondern aus der lateralen Hälfte des Gehirns. Verglichen mit den Tracheaten wăre der Oberlippennerv der Lage seines Ganglions nach mit dem Antennalnerven gleichstellbar.

Aus dem dorsalen Teil des Cerebralganglions entspringt bloss der Opticus der Mittelaugen, welche somit mit den Ocelli der Tracheaten homolog sind. Der Nerv teilt sich alsbald in einen vorderen (op. m) und einen hinteren Ast (op. $\mathrm{m}^{\prime}$ ).

Das somit eng mit dem Gehirn verbundene Ba chmark (bm) beginnt mit einem mächtigen, nach oralwärts $z u$ gerichteten, seinem freien Ende zu zuspitzenden Ganglion, aus dem der Kieferfussnerv an seinem zugespitzten Ende hervorgeht (kfn). Es ist dies das erste Bauchmarksganglion, jenes des Kieferfusses. Mit ihm unkenntlich verwachsen ist das zweite Bauchmarksganglion, aus dem der Nerv (1) des ersten Schreitfusses heraustritt. Die anderen drei Ganglien, die der übrigen Schreitfüsse (2-4), sind zwar mit dem des ersten Schreitfusses sowie unter sich ja auch eng verwachsen, doch jedesmal durch laterale Eindrücke einander gegenüber begrenzt. Aus dem hinteren Ende des letzten Ganglions (4) setzt sich das übrige Bauchmark, jenes des Abdomens (bm') fort.

Die beiden Seitenhälften des cephalothorakalen Bauchmarkes sind miteinander bis zur Unkenntlichkeit ihrer Grenzen von aussen verwachsen und nur ventralwärts findet sich zwischen den beiden Hälften eine ganz tlache Längsrinne (Fig. $1 \mathrm{~B}, \mathrm{bm}$ ), in welcher die Bauchmarksvene verläuft.

Auf der dorsalen Flache des cephalothorakalen Bauchmarkes, dieselbe fast ganz zudeckend, befindet sich die Bauchmarksarterie (Textfig. 1. daj. Sie gibt in jede gangliöse Verdickung dieses Bauchmarksabschnittes, und somit auch für jene des Kieferfusses, je zwei laterale und einen medianen Ast $a b(B)$. Diese verzweigen sich im Bauchmark und bilden dort ein weites grobes Gefässnetz, das sich mit Ästen der ventralwärts gelegenen Bauchmarksvene verbindet. Somit befindet sich im Bauchmarke des Shorpions, wie auch in jenem derjenigen Spinnen, die keinen tracheesierten Cephalothorax haben (4), ein in gewissem Grade geschlossenes Gefässsystem, dessen Wände freilich durchlöchert sein werden. 
Ein ventraler Ast der Aorta (A. ao) gabelt sich dem Darme fest aufliegend über dem cephalothorakalen Bauchmark in zwei Äste, von denen jeder lateral am Darme herabziehend, sich hinter den Cerebralganglien auffallend erweitert (Textfig. $1 \mathrm{~A}, ; 3 \mathrm{da}$ ). Aus jeder dieser Erweiterungen, die nach unten zum Bauchmark ziehen, um dort sich zur beschriebenen Bauchmarksarterie $\mathrm{zu}$ vereinigen, geht ein unterer Ast von hinten in das jederseitige Cerebralganglion (Textfig. 3). Ein oberer Ast zieht am hinteren Rande jedes Cerebralganglions nach oben, etwas mit dem anderseitigen Aste medianwärts $\mathrm{zu}$ konvergierend. Angelangt auf der dorsalen Seite des Gehirns, vereinigen sich die beiderseitigen Äste und dieser unpaare Ast (dg), an der medianen Grenze der beiden Gehirnhälften gelegen (Textfig. 2, dg), stellt die dorsale. Gehirnarterie vor.

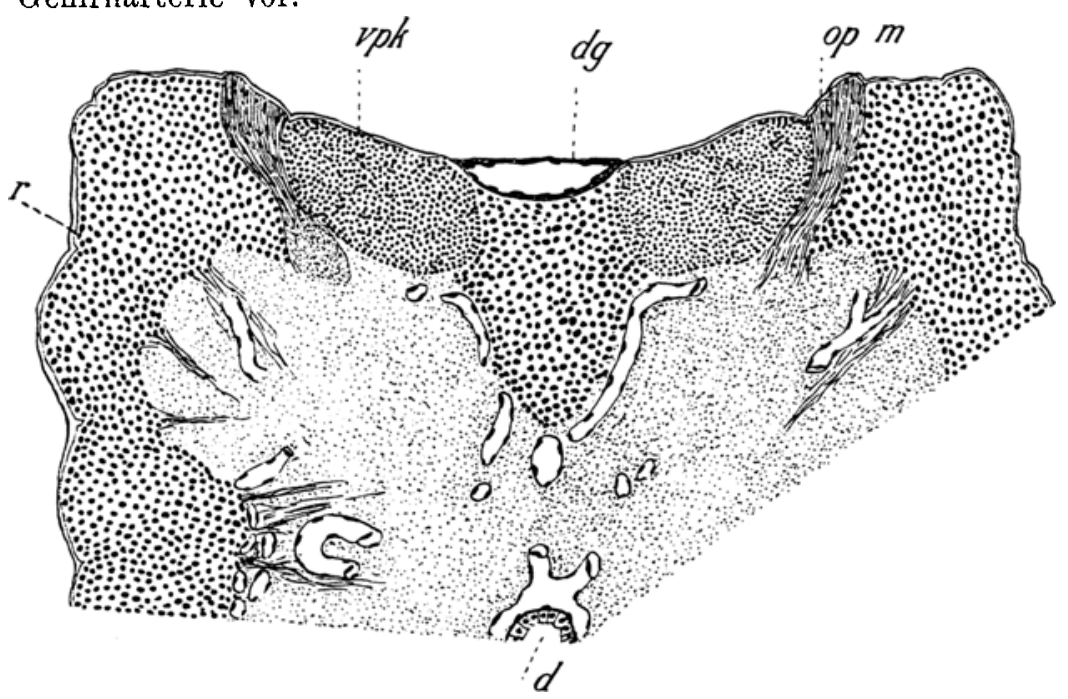

Fig. 2.

Sc orpio e u rop. Querschnitt durch das Gehirn, die beiden vorderen Globuli vpk treffend; $d g=$ Dorsalarterie; opm = Opticus der Medianaugen; $d=$ Darm, $\mathrm{r}=$ laterale Ganglienzellage.

Hier möchte ich nun einiges über den Ba des Gehirns mitteilen, da es uns ja in erster Linie hier auf das Verhalten der Globuli oder pilzhutförmigen Körper ankommt.

Die ganze Ganglienzellage des Gehirns besteht aus gleichgrossen kleinen Zellen (Textfigg. 2, 3), unter denen sich keine grossen Elemente befinden, wie in dem Bauchmarke die grossen 
motorischen Zellen es sind. Von dieser Struktur machen nur je zwei Stellen in jeder Gehirnhalfte eine Ausnahme. Es sind das je zwei ovale Stellen (auf Textfig. $1 \mathrm{~A}$ eingezeichnet) auf der dorsalen Seite jeder Gehirnhälfte, hinter dem Nerven der Scheitelaugen. Diese hintereinander gelegenen Stellen berühren sich nicht. Wie ich noch nachträglich erwähnen will, ist die dorsale Fläche jeder der beiden Gehirnhalften nicht etwa eine der dorsalen Körperoberfläche parallele, sondern ein von vorne nach hinten und unten zu geneigte, wobei in der Längsmitte eine Quermulde sich befindet (Textfig. 3). Durch letztere wird jede Gehirnhälfte in ein vorderes und ein hinteres Stück abgeteilt. Das vordere Stück ist das grössere und endet in einen nach vorne zu gerichteten Hügel, den Apex.

Jene zwei Stellen nun in jeder Gehirnbälfte, die ich als verschieden von der übrigen Ganglienzellenrinde gebaut beschrieben labe, liegen nun so, dass die vordere in der hinteren Hälfte des vorderen Hirnabschnittes (Fig. 3, vpk), die hintere (hpk) in der vorderen des hinteren Hirnabschnittes Platz findet. Medianwärts berühren sich die Paare dieser Stellen nicht, sondern liegt auch zwischen klem vorderen Paar (Textfig. 2, vpk), eine nach innen $z u$ vorspringende Zellenlage der Ganglienzellrinde, über welcher die dorsale Gehirnarterie (dg) hinwegzieht.

Die bezeichneten Stellen sind linsenförmig und bestehen aus kleineren Ganglienzellen als die übrige Hirnrinde und ihre Kerne färben sich intensiver, denn während die des übrigen Gehirns sich mit Alaunkarmin nur dunkelrosa färben, werden diese Zellenkerne tief rotviolett.

Es sind kompakte Zellmassen, ohne dass diese Nervenkerne in ihrer Mitte Faserwerk als Markmasse aufweisen würden, denn eine solche fehlt ihnen vollständig. Es sind diese Kerne die pilzhutförmigen Körper oder Globuli der Skorpione. Dies geht schon daraus hervor, dass sie gerade so wie jene der übrigen Arthropoden je einen nervenfaserigen Stiel (Textfg. 3 st, st') in das Innere des Gehirns entsenden.

Es besitzt somit der Skorpion vier Globuli in seinem Crehirn, ein vorderes und ein hinteres Par, doch steben diese Globuli auf einer sehr niedrigen Stufe ihrer Entfaltung, sie haben sich eben geschieden aus der übrigen Hirnrinde und sind bezüglich ibrer Ent- 
faltung auf etwa gleicher Stufe mit jenen der Myriapoden, wie ich sie seinerzeit beschrieb (2), wenngleich eine dorsale Lage auch schon errungen wurde. Sie besitzen gleich wie

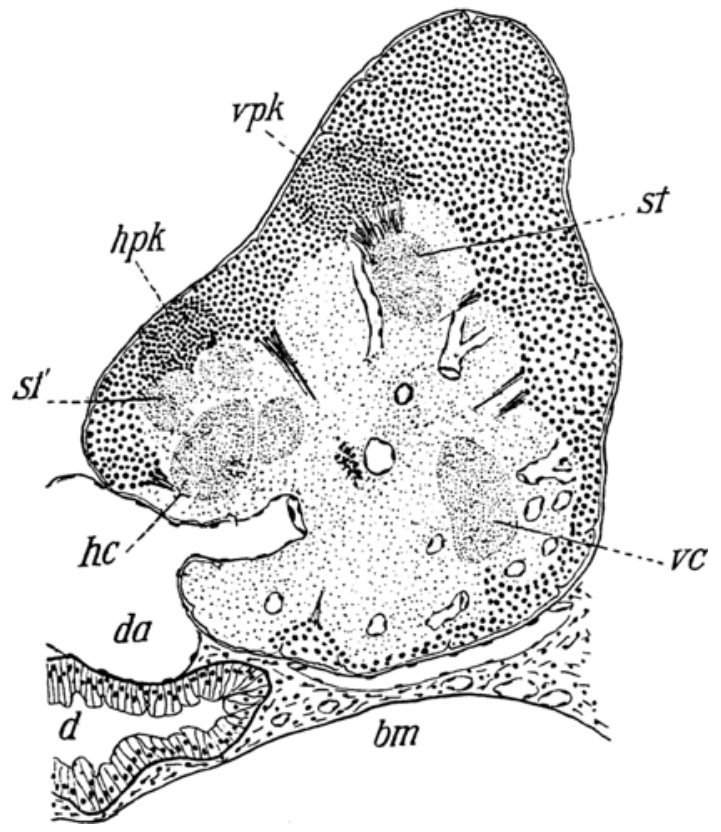

Fig. 3.

Sc orpi o e urop. Sayittalschnitt durch das Gehirn, die beiden Globuli (vpk, hpk) treffend. st' $=$ deren vorderer und hinterer Stiel; $v c=$ vordere, $\mathrm{hc}=$ hintere $\mathrm{Kommissur} ; \mathrm{da}=$ dorsale Bauchmarkarterie, $\mathrm{d}=$ Darm; $\mathrm{bm}=$ Bauchmark.

jene der Myriapoden noch keine Markmasse, und es konnte infolge ihrer geringen Ausdehnung auch zu keinen Faltungen in ihnen kommen.

\section{B. Spinne.}

Über das Zentralnervensystem der Spinnen, deren äussere Form als stark konzentriert schon von altersher bekannt ist, besitzen wir nur eine kurze Mitteilung von Janeck (5).

Nach diesem Autor "sind an dem Gehirne vorn zwei Vorwölbungen, die den Cheliceren zukommen, darüber liegen die breit bis zu den vier grossen Stirnaugen vorspringenden Sehlappen." Besondere Strukturen hat in der Ganglienschichte Janeck nicht gefunden, „mit Ausnahme einiger Verdickungen vorn im Gehirn, 
die man als pilzhutförmige Körper deuten könnte." Vier Lappenbildungen kennzeichnen das Bauchmark der Lycosa amentata nach Janeck "von denen die vier vorderen den Gangbeinen entsprechen und dementsprechend auch die Nervatur für jene Gliedmaßen abgeben." Die Nerven des mittleren hinteren Lappenpaares vereinigen sich ausserdem, soweit sie nicht in die Beine gelangen, jederseits zu einem starken Strange, der in subepithelialer Lage in das Abdomen gelangt.

Neine Untersuchungen beziehen sich auf Epeira und weichen von den Ergebnissen meines Vorgängers so ziemlich ab.

Es liegen die beiden Cerebralganglien (Fig. 1, Cg) gleich wie bei dem Skorpion fest aneinander, da gleich wie dort auch hier sie miteinander innig verwachsen sind, allein ihre dorsale Fläche ist nun nicht mehr nach unten und hinten zu geneigt, sondern ist parallel zur dorsalen Körperoberfläche. An der Seitentläche jeder der beiden Gehirnhälften zeigen sich je zwei hintereinander gelegene Protuberanzen, die bei Lycosa J a n e c $k$ irrtümlich für Sehganglien hielt. Dies sind sie aber nicht und es verlässt das Cerebralganglion der Optikus für die mittleren jederseits zweier Augen der Epeira (op, m) dorsalst und zwischen den beiden Protuberanzen. Er teilt sich sofort in je zwei Äste. Während die hintere Seite der Ganglien allmăhlich und ohne irgendwelche Abgrenzung in das Verbindungsstück mit dem Bauchmark übergeht und steil abfällt, zeigen die Cerebralganglien an der vorderen Seite dem Verbindungsstück gegenüber durch eine quere Einkerbung eine äussere Begrenzung. Dabei kommt äusserlich ein Ganglion für den Oberlippennerv (on) nicht zur Geltung, wie bei dem Skorpion. Diesem Nerven nach innen lagert der Nerv der Seitenaugen (op, l) fest an. Sein Ursprungsgebiet ist auch bier median von jenem Nerven gelegen (Fig. 2, op, l).

Das Ba chmark ist gedrungener als der vordere Bauchmarksabschnitt des Skorpions und entsprechend auch höher. Fünf Ganglien bilden es, wobei das letzte (Fig. 1) durch eine horizontale Einkerbung in eine obere (5) und untere (4) Hälfte gegliedert wird. Das erste Ganglion ist jenes des Cheliceren-Nerven (kfg). Dem Umstand, dass die Chelicere eben nicht jene Mächtigkeit aufweist wie der starke Scheerenfuss des Skorpions, ist es zuzuschreiben, dass auch das Ganglion nicht jenen Umfang besitzt als dort. Er ragt nicht weit nach rorne wie dort, und 
ïberragt das Ganglion des ersten Schreitfusses (1) nicht, sondern ist gleich jenem abgerundet.

So sehen auch die übrigen vier Ganglien aus, die einander gegenüber durch seichte Furchen begrenzt sind.

Das Vernalten des austretenden Nerven ausserhalb des Ganglions habe ich nicht verfolgt und verweise diesbezüglich auf die ausführliche Darstellung Börn ers (1) bei den Pedipalpen.

Vom letzten Ganglion, das ja nun etwas anderes ist wie jenes der Skorpione, da es ja auch das ganze Abdominalmark in sich fasst, treten ausser dem vierten Fussnerven (4) noch die zwei mächtigen übereinander gelegenen Abdominalnerven $a b$.

Der Bau des Bauchmarkes der Spinne, sowie auch des Skorpions, entspricht im wesentlichen jenem Verhalten, das ich für das Bauchmark des Käfers und auch des Regenwurms festgestellt habe (3), d. h. ventralwärts liegt eine hohe Ganglienzelllage, dorsal liegen aber nur spärliche Ganglienzellen (Fig. 4). Auch die Neuroglia spielt jene wichtige Rolle, die ich schon zweimal $(2,3)$ für das Zentralnervensystem der Tracheaten geschildert habe. Sie bildet eine äussere Neurogliahülle um das Zentralnervensystem, deren Elemente bei jenen Spinnen, wie u. a. die Dysderiden sind und bei denen das Bauchmark direkt dem Integumente der ventralen Körperseite anliegt, wie ich schon kurz geschildert habe (4) mit den Epithelzellen durch Fortsätze direkt zusammenhängt.

Diese äussere Hülle sendet nun zahlreiche Fortsätze in die Ganglienzellage, die sich zwischen den Zellen verästeln und so ein Netzwerk bilden, zwischen dessen Maschen die Ganglienzellen liegen. In den Knotenpunkten des Netzes, doch nicht in allen, liegen stark chromophile Zellkerne. Dieses Netz verdichtet sich zwischen der Ganglienzellage und der Markmasse abermals zu einer lockeren, vielfach durchbrochenen Hülle (Fig. 2, 3, 4), von welcher Fortsätze in die Markmasse eindringen, denn auch dort fehlt das kernhaltige Neuroglianetz nicht. Wichtig ist es aber, dass die geschilderte innere Neurogliahülle zwischen Ganglienzellage und Markmasse nach innen zu Fortsätze entsendet, welche um jedes Ganglion herum (Fig. 2, s) eine lockere Hülle bilden. Diese reicht aber nicht in das dorsale Gebiet des Bauchmarkes. Dort treten vielmebr einzelne feinere Fortsätze aus der dorsalen Neurogliahülle in das Mark, dort ein weitmaschiges Netzwerk bildend (Fig. 2, 3, 4 y). 
Auf ein weiteres Verhalten der Neuroglia bei den Spinnen und dem Skorpion, worauf ich schon kurz hingewiesen (4), möchte ich hier aber noch einmal aufmerksam machen. Man sieht dort, wo Gefässe mit ihren Ästen in das Zentralnervensystem eindringen (Fig. 7, g), dass die Wände jener, die wie ein Plattenepithel aussehen, durch Fortsätze direkt mit dem Neuroglianetz des Zentralnervensystems (bm) zusammenhängen. Mit anderen Worten, die Gefässwände werden durch die Verdichtung eines den ganzen Körper durchsetzenden, netzartig zusammenhängenden Stützgewebes (Grobben) gebildet, welches je nach Bedürfnis einer grossen Tascularisation infolge des Riicktritts oder der Nichtentfaltung eines diffiusen Tracheensystems einem grösseren Reichtum von Verästelungen des Blutgefässsystems Vorschub leistet. Bei den Skorpionen, bei denen die primärsten Tracheenbündel sich zu sogenannten Lungen entfaltet haben und dadurch die Atmungsorgane eine strenge Lokalisation erfahren, ist das Gefäßsystem im Zentralnervensystem wenigstens soweit geschlossen, als das immerhin grobe Gefässnetz aus der dorsal gelegenen Arterie in jenes der ventral gelegenen Vene direkt übergeht.

Bei den Spinnen zeigen sich dann sehr verschiedene Grade der peripheren Gefässentfaltung, je nachdem, ob die Tracheesierung der Gewebe - hier insbesondere des Zentralnervensystems eine höhere (Epiblemum u. a.), oder höchste (Dysderiden u. a) ist oder völlig schwindet, wie bei Epeira. Ich verweise diesbezüglich auf meine erste Mitteilung über Arachnoiden (4).

In der Ganglienzellage des Bauchmarkes befinden sich Nervenzellen von sehr verschiedener Grösse, kleine bis sehr grosse. Je kleiner eine Ganglienzelle der Spinnen ist - dies gilt auch für den Skorpion - ist sie um so chromophiler, wodurch die grossen Zellen in der stark tingierten Zellage durch ihre blasse Fürbung auffallen. Die grössten Zellen liegen in jedem Ganglion, wie im Bauchmark überhaupt, an den Abgangsstellen der Nerven, aber hier stets medianwärts (Fig. 3, 4). Es zeigen sich da jedesmal zwei Gruppen solcher grossen Elemente, eine mediale $(\mathrm{m})$ und eine laterale $\left(\mathrm{m}^{\prime}\right)$. Es sind das grosse motorische Zellen - die grössten fand ich beim Skorpion im Ganglion des Kieferfusses -, die peripheren Fasern zum Ursprung dienen. Aus der lateralen Gruppe treten solche Fasern 
direkt in die Nervenwurzel ein und sind somit solche Fasern ungekreuzte, nur für die gleiche Seitenhälfte bestimmt. Doch kann diese Zellgruppe auch gekreuzte Fasern, also für die andere Körperhälfte abgeben (Fig. 4). Die innere Gruppe der grossen Ganglienzellen gibt nur gekreuzte Fasern ab, gleichgültig ob diese zuvor zu Längsfasern werden oder nicht.

Soweit die Faserbündel nicht direkt in die anliegende Nervenwurzel derselben Seitenhälfte eindringen, ziehen sie nach oben und verbinden sich dann, nach innen biegend, arkadenförmig. Dadurch kommt die dorsalwärts lagernde Querkommissur des Bauchmarkes zustande (Fig. 4, vbs), doch schliesst sich dieser dorsalst noch eine andere Ergänzung (v) an, die anderer Herkunft ist. Es umfasst diese Arkade dann medianwärts ein den beiden Bauchmarkshälften gemeinsames Gebiet, das medianwärts in jedem Ganglion durch das senkrecht, gestellte Gefäss durchsetzt wird, welches die Bauchmarksvene $\left(\mathrm{g}^{\prime}\right)$ mit der dorsal gelegenen Arterie (Textfig. 1 B) verbindet. Rechts und links liegen diesem Gefäss je drei Längsbündel übereinander an (Fig. 4, Ib). Diese kommen dadurch zustande, dass entsprechende Nervenbündel aus den Arkadensäulen in sie einbiegen. Auswärts von diesem durch die Arkaden umfassten medianen und unpaaren motorischen Gebiet des Bauchmarkes befindet sich in jedem Ganglienpaar je ein laterales, rein sensorisches Gebiet (a), das somit durch die jeweilige Nervenwurzel und der Arkadensäulen begrenzt wird.

Über den Ursprung der Nerven, sowie den Zusammenhang zwischen dem motorischen und dem sensorischen Bauchmarksgebiet habe ich nun durch die vitale Methylenblaufärbung bei Epeira wenigstens einiges festgestellt, das ich, ohne dabei an eine vollständige Erledigung der Frage zu glauben, hier mitteilen will.

In die Arkaden hinauf ziehen auch Fortsätze von Ganglienzellen (Fig. 4 links, schwarz), die dann oben an der Biegung angelangt. sich in zwei Äste teilen. Der gleichseitige Ast wird zu einer Wurzelfaser für den abgehenden Nerven und biegt in in die gleichseitige Nervenwurzel ein. Der innere Ast durchsetzt die Kommissur, gibt einen Nebenast in das Netzwerk der anderseitigen Halfte des motorischen Gebietes ab und verästelt sich dann in dem sensorischen Gebiet. Doch können Nebenäste 
des Hauptfortsatzes der Zellen auch auf derselben Seite andere Yebenaste und zwar sowohl in das motorische als auch in das sensorische Gebiet abgeben.

Hauptfortsătze anderer Zellen (Fig. 4 rechts, schwarz) gelangen durch die Arkadensäule in die Arkaden, noch auf del gleichen Seitenhälfte des Bauchmarkes sich teilend. Der Nebenast gelangt zur Auflösung in das gleichseitige sensorische Gebiet, wobei. schon früher vom Hauptfortsatz ein Ästchen in dem gleichseitigen motorischen Gebiet sich auflöst. Die Fortsetzung des Fortsatzes aber durchsetzt die Kommissur und gelangt dann als Wurzelfaser in die Wurzel des anderseitigen Nerven.

Das Verhalten anderer aus der Nervenwurzel stammenden Fasern bezieht sich auf einen indirekten Ursprung, aut einen solchen aus dem zentralen Nervennetz. Es sind das Fasern (Fig. 4 rechts), die entweder sich in dem sensorischen Gebiet derselben Seitenhälfte auflösen oder zuvor die Kommissur passierend, dies erst auf der anderseitigen Markhälfte tun.

Ich habe erwähnt, dass der dorsalste Teil der Querkommissur (v) sich anders bildet. In jenem dorsalen Teil des Bauchmarkes (Fig. 2, 3, 4, y), den ich als sehr ganglienzellenarm und hauptsächlich aus einem gröberen Neuroglianetze bestebend, schilderte, befinden sich grosse multipolare Zellen, von denen aber ein Teil zweifellos der Neuroglia angehört. Andere sind ziemlich grosse Ganglienzellen, die sich mit dem grössten Teil ihrer Fortsătze dortselbst auflösen, öfter aber einen längeren Fortsatz durch die Kommissur in die anderseitige Bauchmarkshälfte entsenden. Diese Fasern lösen sich dort im gleichen dorsalen Gebiete auf (Fig. 4 rechts, schwarz). Solche Zellen stehen aber auch mit dem sensorischen Gebiet der gleichen Markhälfte durch kräftigere Fortsätze in Zusammenhang. Da in diesem dorsalen Gebiet sich anch periphere Fasern (Fig. 4 rechts) auflösen, so ist dieses Gebiet als ein sensorisches Dorsalgebiet des Bauchmarkes zu betrachten.

Bezüglich der Längsbahnen des Bauchmarkes steht mir nur ein Befund zur Verfügung. Ich fand da Längsfasern aus einem vorderen Ganglion, im abgebildeten Falle aus dem Ganglion der Cheliceren (Fig. 2, kfg) entspringend, welche das gesamte Bauchmark durchsetzen und in jedes Ganglion je einen Netzfortsatz abgeben. Hier handelt es sich somit um lange Bahnen, 
die alle Ganglien mit dem ersten in Zusammenhang setzen. Solche Verbindungen können aus allen Ganglien abgehen (bei z).

Jene Stelle des Zentralnervensystems, welche jederseits den Ösophagus umgreifend die Kommissuralfasern zwischen Bauchmark und den Cerebralganglien in sich fasst, enthält jederseits die äusserlich nicht wahrnehmbaren Ganglien des Oberlippennerven. Während dann an diesen lateralen Stellen (Fig. 3, r) in der Ganglienzeliage sich auch grosse motorische Zellen befinden, fehlen solche in der vorderen (Fig. 2, r) und hinteren Seite $\left(\mathrm{r}^{\prime}\right)$ dieses den Ösophagus umgreifenden Abschnittes. So verhält sich mit Ausnahme der Globuli die Zellage des Cerebralganglions (Fig. 2, $3 \mathrm{Cg}$ ) auch, denn es bilden sie nur fest aneinandergefügte, gleich grosse aber kleinere Zellen. Innerbalb des Ganglion befindet sich, wie ja auch bei anderen Tracheaten (aber anch bei Branchiaten), eine Vorder- (vc) und eine Hinterkomm is sur (hc). Hinter letzterer und teilweise durch dieselbe bindurch treten Fasern des lateralen Opticus (op. l) nach oben $z u$ in das jederseitige Cerebralganglion, ohne, dass in diesen gerade so wie bei dem Skorpion - irgend eine Stelle als Opticusganglion sich erweisen würde, denn was $J$ an eck als solche bei Lycosa amentata $2 u$ deuten glaubte, jene auch von mir bei Epeira gesehenen Protuberanzen entsprechen entschieden keinen solchen. Vielmehr werden diese, die beim Skorpion fehlen, zum Teil wenigstens durch die mächtiger entfalteten Globuli verursacht. Denn auch die Spinne besitzt in jeder Cerebralganglionhălfte je zwei Globuli, einen vorderen und einen binteren. Infolge ihrer viel mächtigeren Entfaltung als bei dem Skorpion nehmen aber diese Intelligenzspären mehr Platz ein ais dont und sind nun in die vordere (Fig. 1, vpk) beziehentlich hintere Ecke (bpk) des Ganglions verschoben worden. Während dann die hinteren dieser nicht vorspringen, wölben sich die vorderen hügelförmig am Apex yor (Fig. 5). Sie haben jetzt aber auch keine medianwärtige Lage mehr, sondern sind ganz lateralwärts 20 gerückt (Fig. 2, hpk).

Alles dies sind die Folgen Löherer Entfaltung als bei dem Skorpion, denn erreichen die GIobuii der Spinne auch lange nicht jenen hohen Grad als bei den Hymenopteren, geschweige denn bei Limulus, so stehen sie doch etwa auf jener der Orthopteren und haben sich somit von der niederen Stufe der Entfaltung der Myriapoden und Skorpione weit entfernt. 
Sie bestehen aus einer mehrschichtigen kortikalen Ganglienzellage, welche einen ansehnlichen Markkern umgibt, allein nicht allseitig, denn der apikale Teil des Globulus bleibt stets frei von einem Überzug der Ganglienzellschichte (Fig. 2, 5). Der mächtige Stiel (Fig. 3, st) senkt sich tief in den Markteil des jederseitigen Cerebralganglions ein, ohne dass die jeweiligen beiden Stiele medianwärts sich berühren würden.

Die Ganglienzellschichte der Globuli wird von kleinen, fest beisammen liegenden Zellen gebildet, wie überall wo Globuli sich finden. Diese fest beisammen liegenden Zellen stehen untereinander aber in vielfacher Verbindung, wie ich dies für die Tracheaten schon vor Jahren gezeigt habe (2) und nun auch für die Spinne an zwei Stellen der Rinde (Fig. 6, schwarz) nach vitaler Methylenblaufärbung eingetragen habe. An dem faserigen Kern lassen sich zwei Teile unterscheiden: der Apex (ap) und der übrige Kernstiel. Im Apex selbst findet sìch eigentlich nur ein starkes, sehr feinfaseriges Nervenfaserbündel, das hier nach oben ziehend, nach einer Schlingenbildung ( $f$ ) sich wieder in den übrigen Markteil zurückbegibt und von diesem aus dann als der Stiel (st) in die Fasermasse des Gehirns sich versenkt.

Der übrige Faserteil des Globulus wird peripherwärts durch äbnliche Glomeruli (gl und Fig. 5) gebildet, wie wir sie im Antennalganglion besonders der Tracheaten ausführlicher kennen. Der übrige Kernteil, der innere nämlich, wird durch ein feines Nervennetz, aber auch durch gliöse Septen (Fig. 6, nl) gebildet, die oft die ganze Fasermasse durchziehen und vielfach durch ansehnliche Elemente gebildet werden.

Diese Glomeruli sind bei den übrigen Arthropoden unbekannt. Sie kommen auf folgende Weise zustande. Es entsenden Gruppen fest beisammen liegender Ganglienzellen in der Globulusrinde - und hierdurch erscheint diese einigermassen gegliedert - gemeinsame Bündel (Fig. 5), die direkt anf irgend einen der zablreichen Glomeruli gerichtet sind und diesen erreichend, sich in ihn versenken. Auf Methylenblaupräparaten sieht man dann, dass die einzelnen Fasern des Bündels, kräftigere Fortsätze je einer Zelle (Fig. 6) sind die bis zu dem betreffenden Glomerulus hin zahlreiche Nebenästchen abgeben und sich dann ausserhalb der Glomeruli in das Nervennetz des Globulus (m) auflösen. Der Hauptfortsatz mit mehreren, ja vielen seinesgleichen, 
erreicht aber den Glomerulus. Bei der verhältnismässig geringen Zahl dieser ist aber ausgeschlossen die Möglichkeit, dass sämtliche Ganglienzellen der Globulusrinde einen solchen Hauptfortsatz besässen, vielmehr - wie ich es seinerzeit für die Insekten gezeigt habe (2) - sind viele unter diesen Zellen nur solche, die sich mit benachbarten ihresgleichen durch Fortsätze (Interzellularbrïcken) verbinden. Ich bin aber nicht in der Lage, auch nur annähernd die Zahl jener Zellen anzugeben, die mit einem Hauptfortsatze versehen, durch diesen mit einem Glomerulus sich verbinden. Nur das glaube ich, dass in Anbetracht der verschiedenen - die Differenz ist nicht gross - Grösse der Glomeruli nicht immer dieselbe Zahl von Zellen jedem Glomerulus angehört. Nachdem jene Fortsätze den Glomerulus erreicht haben, lösen sie sich dort in ein korbförmiges gröberes Netz auf, aus dessen Maschen wieder aus dem Glomerulus abziehende Nervenfasern entstehen (Fig. 6, gl', gl). Diese Nervenfasern, nun in Bündeln vereinigt, bilden die Schlinge (f) im Apex und nachdem sie sich zu einem gemeinsamen Bündel zusammengetan, verlassen sie als Stiel der Globulus (st) diesen.

Ob innerhalb des Glomerulus jede Nervenfaser oder Ganglienzellfortsatz sein eigenes eingeschaltenes Netz, also Nebenglomerulus im Hauptglomerulus besitzt, vermag ich nicht zu unterscheiden, obgleich es öfter den Anschein hat, wie wenn dem so wäre. Jedenfalls sind diese eingeschobenen Netze oder Nebenglomeruli, mögen sie nun in Einzel- oder gemeinsamen Gilomerulus sein, dazu angetan sein, die Stromstärke in der leitenden Faser durch Resistenz zu mehren. Ich erinnere nur an die Tatsache, dass wenigstens (2) bei manchen Myriapoden, der einfache Globulusstiel schraubenförmige Windungen eingeht, die in Ermangelung von Glomeruli dasselbe bezwecken werden.

Es treten aber mit dem Stiel auch solche Fasern in den Kernteil des Globulus, die sich gleich in demselben auflösen ohne mit Ganglienzellen dort oder den Glomeruli irgend in eine nähere - unvermittelte - Beziehung zu treten. Es sind das Fasern, die aus oft weiten Nervengebieten hierher in den Globulus gelangen also globipetal leitende Elemente (n).

$\mathrm{Ob}$ ich saimtliche Verbindungen der Globusstiele ermittelt habe, wäre zu bezweifeln, ich beschränke mich darum nur darauf, was ich bestimmt erkennen konnte. 
Aus dem Stiel des Globulus treten Fasern in das Schlundkommissurenbündel (Fig. 3 rot), die entweder anf derselben Seitenhälfte im Bauchmark oberhalb der ventralen Ganglienzellage sich verästeln oder durch die Bauchmarkkommissur hindurchziehend, an gleicher Stelle der anderen Seite enden. Andererseits kommen kollaterale Äste sensibler peripherer Nervenfasern (rechts) bis in den gleichseitigen Stiel und geraten mit diesem entweder in den gleichseitigen Globulus oder treten durch eine dorsalwärtige, sehr geringe Kommissur unter der Ganglienzellschichte der dorsalen Cerebralganglienseite hinüber in den anderseitigen Globulus. Durch diese Kommissur hindurch treten aber auch - wie eingezeichnet - Fasern aus dem anderseitigen Globulus in den betreffenden Globulus.

Nebenbei möchte ich bemerken, dass oberhalb jener erwähnten Kommissur, zwischen ihr und der Ganglienzellage des Gehirns eine schmale Lage von Fasermasse sich findet (cg), welche ihrer Lage nach nur als die Vorstufe des Zentralganglions der Tracheaten gelten könnte. $\mathrm{Zu}$ einer Einwanderung von Ganglienzellen aus der darüber gelegenen Zellage ist es aber nicht gekommen.

Eine Verbindung der Globuli mit Fortsätzen von motorischen Ganglienzellen des Bauchmarkes besteht gleichfalls, allein mir ist nur der folgende Fall bekannt geworden. Es entsandte eine Ganglienzelle des Bauchmarks durch das gleichseitige Schlundkommissurenbündel einen langen Fortsatz nach oben (Fig. 3, links). Dieser Fortsatz liess einen Nebenast in die vordere Cerebralkommissur (vc), einen anderen in die Fasermasse des Gehirns gelangen und begab sich dann durch den Stiel des gleichseitigen Globulus in denselben.

Festgestellt wäre somit, dass die Globuli der beiden Seitenhälften nicht nur untereinander, sondern auch mit motorischen und sensorischen Bauchmarkbezirken in Verbindung stehen und zwar mit ersteren sowohl durch globulipetal als durch globulifugal leitende Fasern.

Verbindungen der dorsalen Cerebralganglienzellage mit motorischen Bauchmarkbezirken sah ich auch (Fig. 3, rechts). Hier handelt es sich wohl um die Bezirke der mittleren Ocellen. 
Das Wesentlichste was in vorliegender Schrift ïber die Globuli der Spinne festgestellt werden konnte, ist somit, dass diese eine weit böhere Stufe der Entfaltungerreicht haben als jene der Skorpione, sie dürften diesbezüglich mit den Orthopteren sich messen oderdenendoch gleichkommen. Die Differenz zwischen dem Entwicklungsgrad der Globuli der Skorpione und der Spinnen ist so gross, wie jene zwischen dem der Myriapoden und niederster Hexapoden einerseits und etwa der Orthopteren andererseits.

Lnd dies ist das Hauptergebnis vorliegender Studie, woran ich nun den Vergleich der Entfaltung der Limulusglobuli mit jenen der skorpione und Spinnen anknüpfen will, wobei ich auf das völlig verschiedene Ferhalten des gesamten Nervensystems von Limulus gleich jetzt schon hinweisen möchte. Hierbei halte ich mich an die Angaben Viallanes (9), welche ausführlicher als

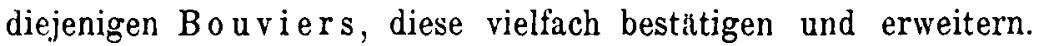

Es besteht das Zentralnervensystem von Limulus aus einem Supraösophageal-Ganglion, dem Gehirn also und dem langen Bauchmark, das aus vielen Ganglienpaaren besteht, die durch Querkommissuren miteinander zusammenhängen. Hierbei zeigt das vordere Bauchmarkende eine selbständige Konzentration drei paarer Ganglien, wie denn überall bei Branchiaten und Tracheaten, aber nirgends bei Arachnoiden, wie ich dies gleich hervorheben möchte. Die Konzentration des vorderen Bauchmarkes zu einem solchen Stücke, wie es die Skorpionen aufweisen und in welches bei der Spinne auch das Abdominalmark mit einbezogen war, kam es hier also nicht. Der Nerv der Cheliceren entspringt aus dem vordersten Ende des Bauchmarkes, das bereits zum Subösophageal-Bezirk gehört. Eine Homologisierung der Cheliceren mit Antennen wäre somit, wie ich bemerken möchte, auch hier ausgeschlossen. An dem Gehirn des Limulus fallen die sehr umfangreichen Globuli, die „corps pédoncolés" auf, die auch Viallanes in gleicher Weise auffasst. Über sie berichtet unser Autor, dass sie eine verzweigte Form batten und weiter, dass: „l'extrémité inférieur de la tige senfonce dans la substance du lobe protocérébral correspondant; l'éxtrémité superieure se divise dichotomiquemente en un grand nombres des branches. Ces dernièrs, qui se terminent par des extrémités arrondies, sont formées d'une substance ponctuée à trame très 
fine, et entièrment revêtues par des noyaux ganglionnaires, semblables a ceux qui revêtent les colps pédonculés des Insectes. Les corps péduncules sont plus developpés chez le Limule que chez aucun autre Arthropode; ils enveloppent la totalité du cerveau, en ne laissent libre qu'une partie des ganglions optiques et ocellaires; leur volume est considérable, et i eux seuls il constituent presque toute la masse cérébral." (l. c. pag. 416, 417). ${ }^{1}$ )

Hiermit begnüge ich mich, denn es wurde nicht nur der grosse Linterschied im gesamten Zentralnervensystem des Limulus und der Skorpione gezeigt, sondern auch weiter oben darauf hingewiesen, dass bei diesen und den Spinnen ein Homologon der Tracheatenantenne in den sog. Oberlippenfortsätzen besteht.

Hauptsächlich ist es aber das Verhalten der Globuli, welches ein Ableiten der Skorpione von Limulus auch bezüglich des Zentralnervensystems illusorisch macht. Denn ware dem so. und stünde der Limulus irgend in einer Beziehung zu Vorgăngernder Skorpione, so müssten diese doch die mächtige Entfaltung der Globuli geerbt haben oder mindestens höher entfaltete Globuli besitzen, als die niedersten Articulaten. Dem ist aber nicht so, denn siestehendiesbezüglich auf jenerniedrigen Stufe, der gegenüber jene der Spinnen als jüngerer Formen des Stammes, eine höhere Entfaltung aufeist.

Der Einwand aber, dass bei den Skorpionen sich möglicherweise diesbezüglich um Rückbildungserscheinungen handeln würde - wohl ein schwaches Argument - wird dadurch zunichte, dass hier weder Parasitismus noch irgend eine ersetzende Einrichtung zur Hand ist. So einen Ersatz habe ich bekanntlich in den höheren Komplexaugen bei den Tracheaten festgestellt.

Auch nach Vollendung dieser Studie bleibe ich somit bei meiner Aussage in meiner früheren Arbeit über die Atmungsorgane der Arachnoiden. Diese lautet: „Büschelförmige Tracheenpaare in gleichmässiger Anordnung im segmentiertem Körper der Arachnoidenvorfahrell, wofür chilopode Myriapoden noch Zustånde aufweisen - ohne als Arachnoidenahnen zu gelten - waren

1) Hätte Viallanes die diesbeziiglichen Verbältnisse bei den Skorpionen und Spinnen gekannt, so hätte er sich kaum dazu hinreissen lassen, den Limulus im Ray-Lankester schen Sinne den Cheliceraten einzuverleiben. 
die Anfangsstufen, von wo aus mit der Umformung des Körpers viele Tracheen-Paare zugrunde gingen, mindestens vier, wofür die Skorpione eintreten. sich aber erhielten. Welche Paare diese in jedem Arachnoidenfalle nach der Segmentreihe sind, entzieht sich heute der Beurteilung. Von diesen vier Paaren erhielten sich im besten Falle bei der weiteren Phylogenese aber nur drei, demn bei Wilben sowohl als bei den Phalangiden sind im höchsten Falle nur zwei Paare nachweisbar. Dabei erhöht sich selbst bei der höchsten Verzweigung dieser Tracheen im Gegensatze zu den Tracheaten (Myriapoden und Hexapoden) die feste Tendenz der Isolierung nicht nur der einzelnen Tracheen derselben Seitë voneinander: sondern auch die von jenen auf der anderen Körperhälfte. Wir kennen nur eine Querverbindung, eben im Vordertracheensystem der Spinnen, die aber als sekundär erworben und nicht als ererbt $\mathrm{zu}$ betrachten ist. Entweder entfalteten sich alle gebliebenen Tracheenpaare zu Lungen (Skorpione) oder nur zwei oder sogar bloss ein Paar oder gar keines (Caponiden). Bei der Entfaltung von vier Lungen wurde das dritte Tracheenpaar aufgehoben, die Lungen ersetzen das übrige, oder aber es erhält sich ausser einem Lungenpaar bei den Spinnen noch ein Tracheenpaar. Aber auch dafür haben wir ja Beispiele, dass auch bei einem Lungenpaar alles übrige von Atmungsorganen in Wegfall gerät wie bei einem Teil der Pedipalpen. Bei einem anderen Teil der Arachnoiden gelangt es aber gar nicht zur Lungenentfaltung. Ein völliges Schwinden konzentrierter Atmungsorgane ist aber ein Zustand, der mit Ausnahme der Chordaten - da bei Salamandrina und Spelerpiden der Kopfdarm und Ösophagus noch auch Atmungsorgan ist - sich bei allen Bilaterienabteilungen einstellen kann."

Heidelberg, im November 1911. 


\section{Literaturverzeichnis.}

1. B ̈̈rner, C.: Zur Kenntnis der Pedipalpen. Zoologica, Bd. XVII, 1904.

2. $\mathrm{H}$ a 1 l e r, B. : Über den allgemeinen Bauplan des Tracheatensyncerebrums. Arch. f. mikr. Anat., Bd. LXV.

3. Derselbe: Über das Bauchmark. Jenaische Zeitschr. f. Naturwiss ; Bd. XLVI.

4. Derselbe: Über die Atmungsorgane der Arachnoiden. Ein Beitrag zur Stammesgeschichte dieser Tiere. Arch. f. mikr. Anat., Bd. LXXIX, Abt. I.

5. Ja neck, R.: Das Gehirn und Bauchmark der Spinnen. Verhandl. d. Gesellschaft deutscher Naturforscher und Ärzte. 82. Versammlung. Königsherg 1910.

6. Newport: G.: On the structure, relation and developement of the nerous and circulatory systems in Myriapoda and macrourons Arachnida. Philos. Transact., 1843.

7. R a y - Lankester, E.: Limulus an Arachnid. Quart. Journ. for Wikrosk. Sc., V. XXIII, 1880.

8. Derselbe: The Structure and Classification of the Arachnoida. Ebendort, V. XLVIII, 1905.

9. Vi allanes, M. H.: Centres nerveux et les organes des sens des animanx articulés. Ann. Sc. nat.: Ser. VII, T. XIV, 1892.

\section{Erklärung der Abbildungen auf Tafel XXVI.}

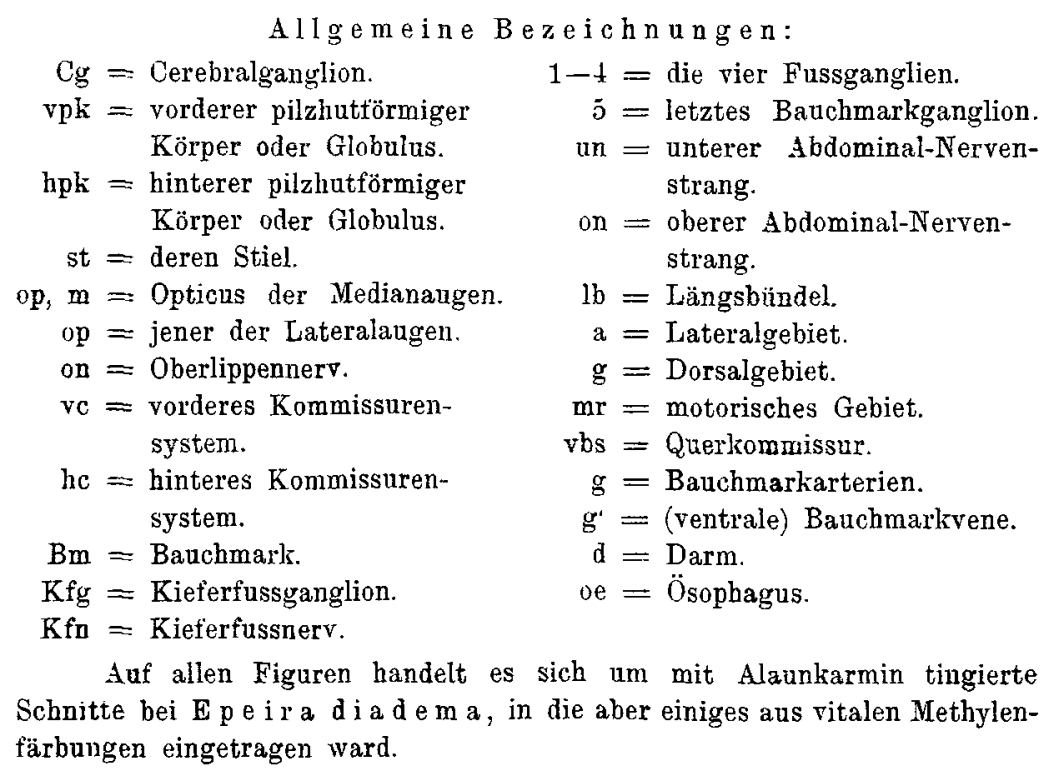


524 B. H a ller: Das Zentralnervensystem des Skorpions etc.

Fig. 1. Das ganze Zentralnervensystem von der rechten Seite. Aus dem Körper herausgeschältes Totalpräparat.

Fig. 2. Sagittaler Längsschnitt, die Globuli treffend.

Fig. 3. Querschnitt, die hinteren Globuli treffend, durch Gehirn und Bauchmark.

F'ig. 4. Querschnitt durch das dritte Bauchmarkganglion, die abgehenden Nerven treffend.

Fig. 5. Schräg von vorne nach hinten gerichteter Querschnitt durch die vorderen Globuli.

Fig. 6. Querschnitt durch den linken vorderen Globulus. $\mathrm{Gl}=$ Glomeruli; $\mathrm{rd}=$ Rinde; $\mathrm{m}=$ Mark; ap = Apex; $\mathrm{nl}=$ neurogliales Septum.

Fig. 7. Epiblemum salticum. Ein Stück aus einem sagittalen Längsschnitte. $\mathrm{d}=$ Darm; $\mathrm{g}=$ Gefäss; bm = Bauchmark. 


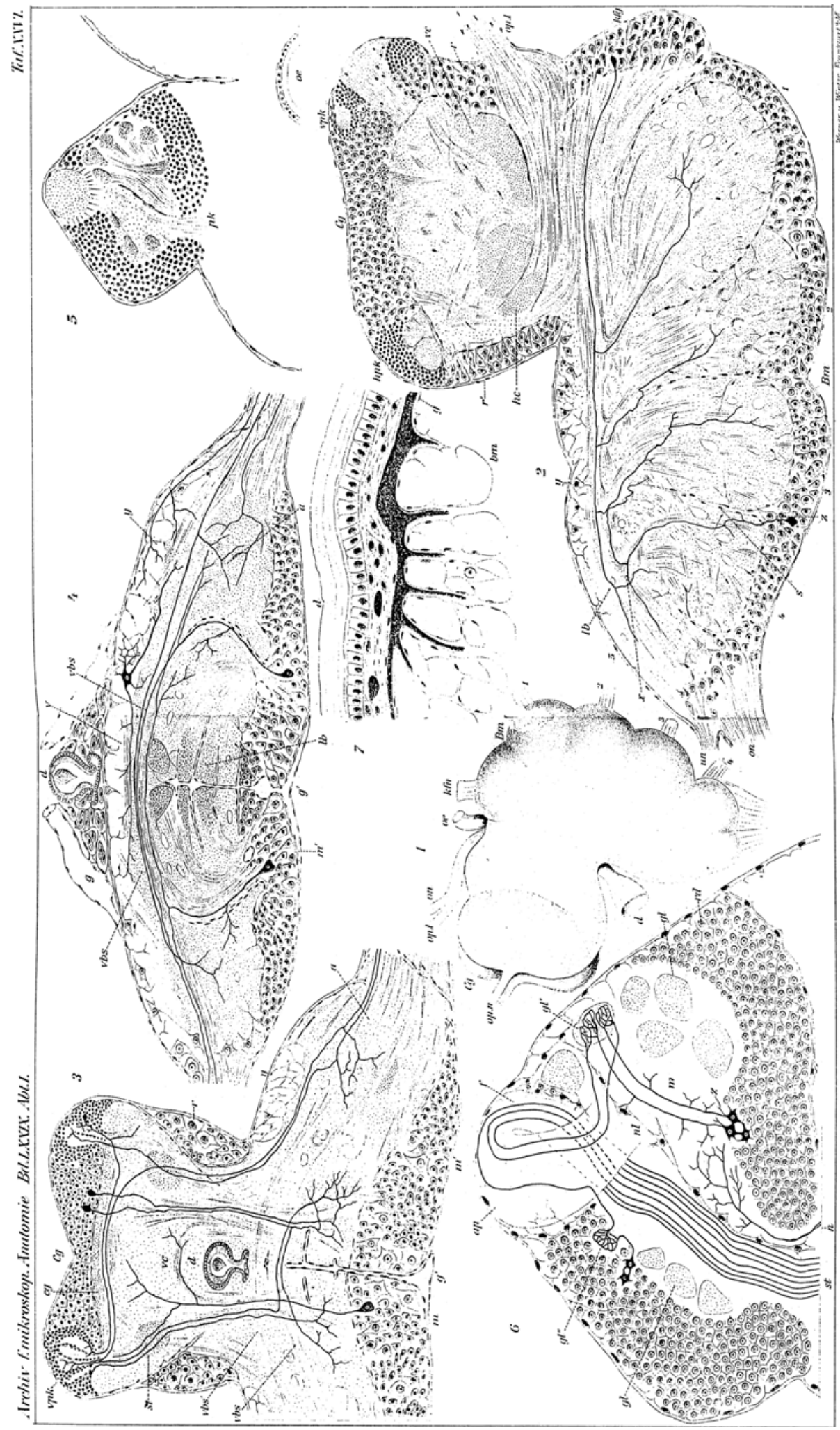

